\title{
Circulating miR-199a-3p as a novel serum biomarker for colorectal cancer
}

\author{
RYOJI NONAKA ${ }^{1}$, JUNICHI NISHIMURA ${ }^{1}$, YOSHINORI KAGAWA ${ }^{1}$, HIDEKI OSAWA ${ }^{1}$, JUNICHI HASEGAWA ${ }^{2}$, \\ KOHEI MURATA ${ }^{3}$, SHU OKAMURA ${ }^{3}$, HIROFUMI OTA ${ }^{4}$, MAMORU UEMURA ${ }^{1}$, TAISHI HATA ${ }^{1}$, \\ ICHIRO TAKEMASA ${ }^{1}$, TSUNEKAZU MIZUSHIMA ${ }^{1}$, DAISUKE OKUZAKI ${ }^{5}$, \\ HIROFUMI YAMAMOTO ${ }^{1}$, YUICHIRO DOKI ${ }^{1}$ and MASAKI MORI ${ }^{1}$ \\ ${ }^{1}$ Department of Surgery, Gastroenterological Surgery, Graduate School of Medicine, Osaka University, Osaka; \\ ${ }^{2}$ Department of Surgery, Osaka Rosai Hospital, Osaka; ${ }^{3}$ Department of Surgery, Suita Municipal Hospital, Osaka; \\ ${ }^{4}$ Department of Surgery, Saiseikai Senri Hospital, Osaka; ${ }^{5}$ Special Research Facilities, DNA-chip Development \\ Center for Infectious Diseases, Research Institute for Microbial Diseases, Osaka University, Osaka, Japan
}

Received March 31, 2014; Accepted July 7, 2014

DOI: $10.3892 /$ or.2014.3515

\begin{abstract}
Serum microRNAs (miRNAs) have been shown to have potential for cancer diagnosis. The main objective of the present study was to identify a novel serum miRNA biomarker from patients with colorectal cancer (CRC). Microarray analysis of miRNA expression was performed using paired pre-operative and post-operative serum from $10 \mathrm{CRC}$ patients. Expression of two miRNAs (let-7a and miR-199a-3p) was significantly decreased in the post-operative serum when compared to levels in the pre-operative serum $(\mathrm{P}=0.015$ and 0.029 , respectively). Quantitative real-time polymerase chain reaction (qRT-PCR) confirmed the decrease in the miRNAs in an extended number $(n=30)$ of paired serum samples. Next, we examined the serum let-7a level in 32 non-cancer patients and $84 \mathrm{CRC}$ patients but we found no significant difference $(\mathrm{P}=0.120)$. In contrast, miR199a-3p expression was significantly higher in the CRC patients than that in the non-cancer patients $(\mathrm{P}=0.016)$. Furthermore, clinical and pathological survey indicated that high expression of miR-199a-3p was significantly associated with deep wall invasion. Our data suggest that circulating miR-199a-3p could be a novel serum biomarker for CRC.
\end{abstract}

Correspondence to: Dr Hirofumi Yamamoto, Department of Surgery, Gastroenterological Surgery, Graduate School of Medicine, Osaka University, 2-2 Yamadaoka, Suita City, Osaka 565-0871, Japan

E-mail: hyamamoto@gesurg.med.osaka-u.ac.jp

Abbreviations: CRC, colorectal cancer; miR/miRNA, microRNA; qRT-PCR, quantitative reverse transcription-polymerase chain reaction

Key words: miR-199a-3p, circulating miR, colorectal cancer

\section{Introduction}

Colorectal cancer (CRC) is one of the leading causes of cancerrelated mortality worldwide. In spite of screening examination, CRC incidence and the associated mortality have increase rapidly in the past several decades $(1,2)$. The prognosis of CRC is associated with stage, and CRC can be completely cured if detected early; the 5-year survival rate is $93.2 \%$ for stage $\mathrm{I}$, and $8.1 \%$ for stage IV (3). Thus, early detection of CRC is crucial to reduce mortality, and CRC-related deaths can be prevented through early detection and early treatment. Several CRC screening tests including fecal occult blood testing (FOBT) and colonoscopy have been available for years (4). However, these methods are associated with issues such as low adherence rates, high cost or low sensitivity. An ideal screening method should have a high sensitivity and specificity for early stage CRC; it should also be safe and accepted by patients (5-7).

MicroRNAs (miRNAs) are 22-nucleotide non-coding RNA molecules that regulate a variety of cellular processes including cell differentiation, cell cycle progression and apoptosis. miRNAs have been demonstrated to play an important role in the multistep processes of carcinogenesis either by oncogenic or tumor-suppressive function. The study of miRNAs has been extended to many types of tumors, including CRC (8-10). These studies have revealed that miRNAs may be potential diagnostic or prognostic tools for human cancers.

Tumor-associated RNAs have been described in the serum/plasma of cancer patients for more than a decade. More recently, several studies have also demonstrated that circulating miRNAs exist in serum/plasma $(11,12)$. Accordingly, several subsequent studies have proven that miRNAs can serve as potential biomarkers for various diseases including cancer. It has been revealed that miR-92 and miR-21 are significantly elevated in the plasma of CRC patients and can be potential non-invasive molecular markers for CRC detection (13-16). In the present study, we aimed to identify a novel circulating miRNA in CRC patients and to evaluate its feasibility as a noninvasive diagnostic test for efficient detection of CRC. 


\section{Materials and methods}

Patients and samples. Informed consent was obtained from CRC patients and non-cancer patients for the use of their blood samples. From April 2011 to June 2013, venous blood samples were collected from the CRC patients $(n=114)$ and non-cancer patients who suffered inguinal hernia or gall bladder stone $(n=32)$. In 30 of the CRC patients, blood samples were obtained before and on the 7th day after surgery. No cancer patients received chemotherapy or radiotherapy before blood sampling. The blood samples were obtained from Osaka University, Osaka Rosai Hospital, Suita Municipal Hospital and Saiseikai Senri Hospital. Whole blood was collected, centrifuged at $1,000 \mathrm{rpm}$ and $4^{\circ} \mathrm{C}$ for $15 \mathrm{~min}$. The supernatant fluids were centrifuged at $15,000 \mathrm{rpm}$ and $4^{\circ} \mathrm{C}$ for $10 \mathrm{~min}$. The supernatant fluids were stored at $-80^{\circ} \mathrm{C}$ until RNA extraction. This study was conducted under the supervision of the Ethics Board of Osaka University Hospital.

RNA extraction. Small RNA was enriched from all serum samples using the mirVana Paris RNA isolation kit (Ambion, Austin, TX, USA), following the manufacturer's instructions. Briefly, $400 \mu \mathrm{l}$ of serum was thawed on ice and centrifuged at $15,000 \mathrm{rpm}$ for $15 \mathrm{~min}$ to remove cell debris. Next, $300 \mu \mathrm{l}$ of the supernatant was lysed with an equal volume of $2 \mathrm{X}$ denaturing solution. For normalization of sample-to-sample variation during the RNA isolation procedures, $20 \mathrm{fmol}$ of synthetic $C$. elegans miRNA cel-miR-39 was added to each denatured sample. Small RNAs were then enriched and purified following the manufacturer's protocol. The concentration of all RNA samples were quantified by NanoDrop ND-1000 (Nanodrop, Wilmington, DE, USA).

miRNA microarray analysis. miRNA microarray experiments were carried out using Agilent human miRNA microarray catalogued in the Sanger database ver. 12.0 (design ID 021827). Approximately $10 \mathrm{ng}$ of aliquots of total RNA with cel-miR-39 was used for making the miRNA probes according to the Agilent protocol (ver. 2.3). Microarrays were performed for paired pre-operative and post-operative serum from $10 \mathrm{CRC}$ patients. Briefly, total RNA was dephosphorylated with calf intestine alkaline phosphatase, denatured with dimethyl sulfoxide, and labeled with pCp-Cy3 using T4 RNA ligase using an miRNA labeling reagent and hybridization kit. Probes were hybridized at $55^{\circ} \mathrm{C}$ for $20 \mathrm{~h}$ with rotation. Then the slides were washed by Gene Expression Wash Buffer 1 at room temperature for $5 \mathrm{~min}$ and by Gene Expression Wash Buffer 2 at $37^{\circ} \mathrm{C}$ for $5 \mathrm{~min}$. After hybridization and washing, the slides were scanned using an Agilent scanner (G2505C). Images were extracted using Agilent Feature Extraction software (ver. 10.7.3.1) and Agilent GeneSpring GX software (ver. 10.0.2). Differences in miRNA expressions between the 10 pairs was determined when the fold-change of cel-miR-39 normalized expression values was $>2.0$ and the $\mathrm{P}$-value was $<0.05$ using paired t-test for further analysis. The microarray raw data are available in Gene Expression Omnibus (GEO;http://www.ncbi.nlm.nih. gov/geo) under accession no. GSE ${ }^{* * * * *}$.

$q R T-P C R$. For the microRNA-based RT-PCR assays, $2.5 \mu 1$ of enriched small RNAs from serum samples was reverse

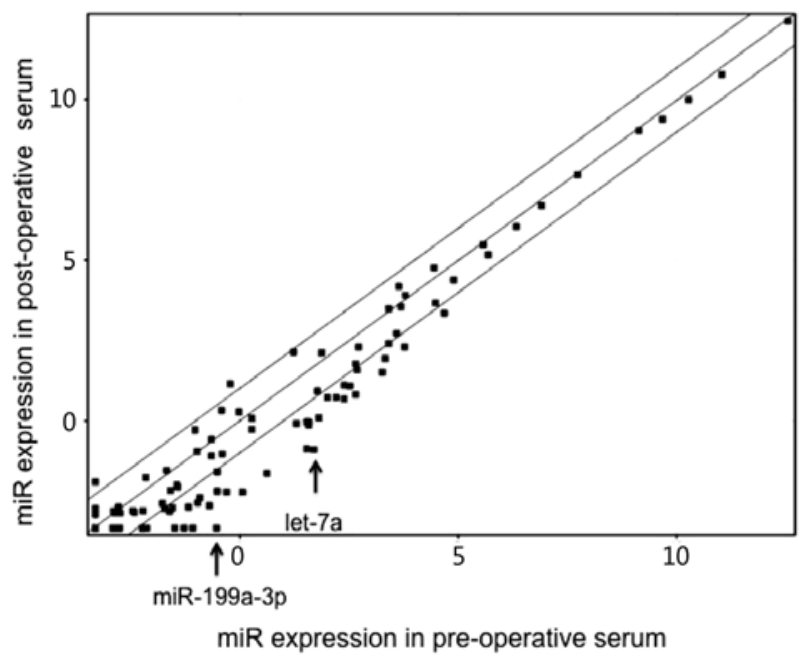

Figure 1. Results of the miRNA array analysis. Following comparison of the pre-operative and post-operative patient serum $(n=10)$ by miRNA microarray analysis, expression of two miRNAs (let-7a and miR-199a-3p) was decreased in the post-operative serum as compared to the pre-operative serum $(\mathrm{P}=0.015$ and 0.029 , respectively). The arrows indicate miR-199a-3p and let-7a.

transcribed using the TaqMan MicroRNA Reverse Transciption kit (Applied Biosystems, San Diego, CA, USA) according to the manufacturer's instructions in a total reaction volume of 7.5 $\mu 1$. A 1:20 dilution of RT products was used as template for the PCR stage. PCR reaction was performed in triplicates using TaqMan 2X Universal PCR Master Mix according to the manufacturer's instructions. Each reaction was performed in a final volume of $20 \mu \mathrm{l}$ containing $1.33 \mu \mathrm{l}$ of the cDNA and $1 \mu \mathrm{l}$ of TaqMan miRNA Assay Mix. The amplification profile consisted of denaturation at $95^{\circ} \mathrm{C}$ for $10 \mathrm{~min}$, followed by 40 cycles of $95^{\circ} \mathrm{C}$ for $15 \mathrm{sec}$ and $60^{\circ} \mathrm{C}$ for $60 \mathrm{sec}$. Each sample was run in triplicates for analysis. The cycle threshold $(\mathrm{Ct})$ is defined as the number of cycles required for the fluorescent signal to cross the threshold in qPCR. The 7900 Sequence Detection System 2.3 (Applied Biosystems) software was used to compute the relative change in RNA expression by the $2^{-\Delta \Delta \mathrm{Ct}}$ method with $95 \%$ confidence intervals.

Primers. The miRNA-specific primer sequences, including miRNA-199a-3p, let-7a, cel-miR-39 and RNU6B, were designed based on the miRNA sequences obtained from the miRBase. The primer sequences were: hsa-miR-199a-3p, 5'-ACAGUA GUCUGCACAUUGGUUA-3'; hsa-let-7a, 5'-UGAGGUAGU AGGUUGUAUAGUU-3'; hsa-miR-21, 5'-UAGCUUAU CAGACUGAUGUUGA-3'; cel-miR-39, 5'-UCACCGGGU GUAAAUCAGCUUG-3' and RNU6B, 5'-CGCAAGGATG ACACGCAAATTCGTGAAGCGTTCCATATTTTT-3'.

Statistical analysis. The significance of the serum miRNA level was determined by Mann-Whitney, Wilcoxon and $\chi^{2}$ tests where appropriate using the GraphPad Prism 6 (San Diego, CA, USA). The sensitivity, specificity and accuracy were calculated according to standard formulas. Receiver operating characteristic (ROC) curve and area under the ROC curve (AUC) were established for discriminating patients with CRC. P-values of $<0.05$ were considered to indicate a statistically significant result. 

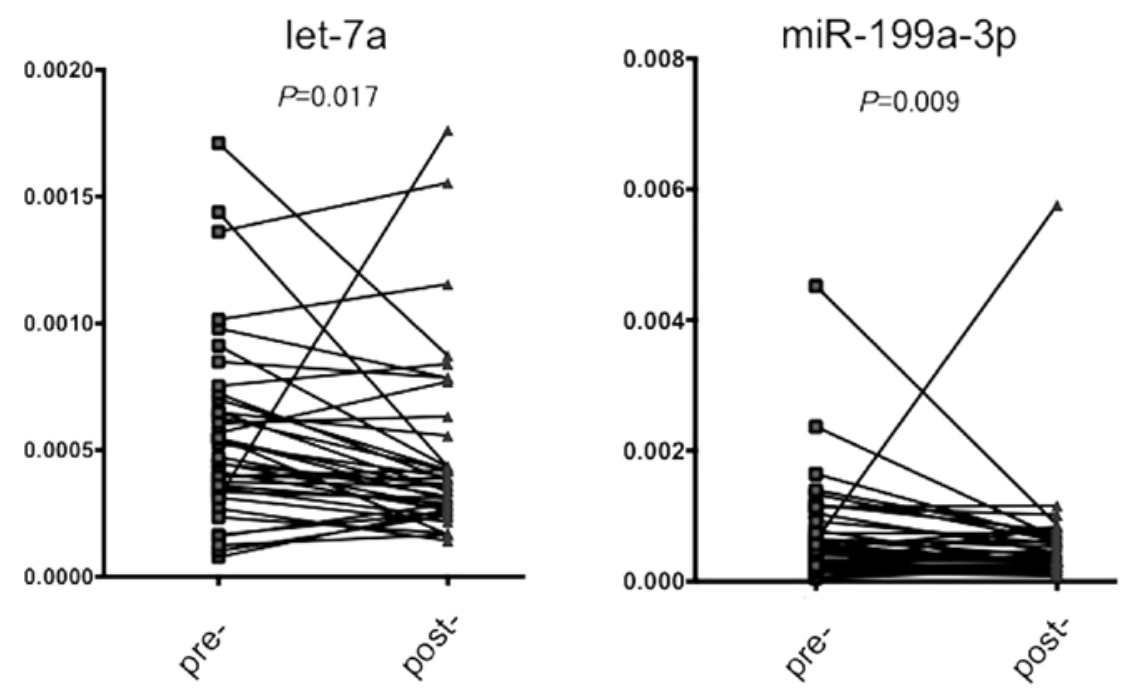

Figure 2. Confirmation of the results obtained from the miRNA array by qRT-PCR. We confirmed the results obtained from the miRNA array by qRT-PCR in serum samples of CRC patients $(\mathrm{n}=30)$. A significant decrease in miRNA levels was noted in the post-operative serum when compared with the pre-operative serum for both miRNAs; $\mathrm{P}=0.017$ for let-7a and 0.009 for miR-199a-3p, respectively. miRNA, microRNAs; CRC, colorectal cancer.


\section{Results}

Results of the miRNA microarray analysis. Following comparison between the pre-operative and post-operative CRC patient serum ( $\mathrm{n}=10 ; 4$ stage II CRCs and 6 stage III CRCs) by the miRNA array, we identified miRNAs, the majority of which showed a decrease in the post-operative serum (Fig. 1). Among them, we focused on two miRNAs, miR-199a-3p and let-7a, whose expression showed the largest decrease after surgery with significant P-values (6.82- and 5.92-fold decrease; $\mathrm{P}=0.015$ and 0.029 , respectively, Fig. 1).

Confirmation of the results obtained from the miRNA array by $q R T-P C R$. We then attempted to confirm the results obtained

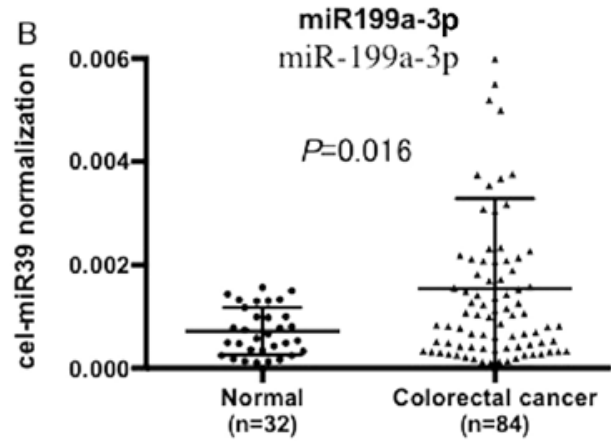

Figure 3. Comparison of the serum miRNAs in non-tumor $(n=32)$ and CRC patients $(n=84)$. (A) Serum let-7a expression in non-tumor and CRC patients. There was no significant increase in serum let-7a in the CRC patients $(\mathrm{P}=0.120)$. (B) Serum miR-199a-3p expression in non-tumor and CRC patients. A significant increase in $\mathrm{CRC}$ patients was noted $(\mathrm{P}=0.016)$ (C) ROC curve was drawn for serum miR-199a-3p, which yielded 0.644 as the AUC value. When a cut-off point was set at 0.0010 , the sensitivity was $47.6 \%$ and the specificity was $75.0 \%$ in discriminating CRC from non-tumor control subjects. miRNA, microRNAs; CRC, colorectal cancer.

from the miRNA array by qRT-PCR in extended samples of CRC patients ( $\mathrm{n}=30)$. As shown in Fig. 2, a significant decrease in miRNA levels was noted in the post-operative serum for both miRNAs ( $\mathrm{P}=0.017$ for let-7a and 0.009 for miR-199a-3p, respectively), suggesting that the expression levels of these miRNAs were reduced after removal of the main tumor by surgery.

Expression of miRNAs in the serum of normal and CRC patients. We examined the serum let-7a level in 32 non-cancer patients and $84 \mathrm{CRC}$ patients and no significant difference was noted ( $\mathrm{P}=0.120$, Fig. 3A). In contrast, miR199a-3p expression was significantly higher in the CRC patients than that in the non-cancer patients ( $\mathrm{P}=0.016$, Fig. $3 \mathrm{~B})$. An ROC curve was 

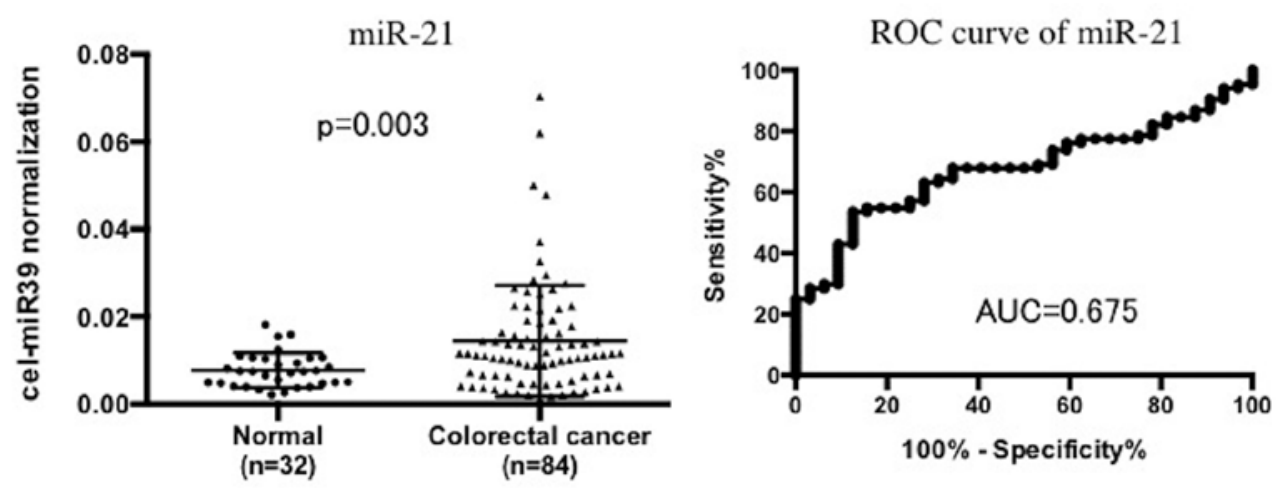

Figure 4. Serum miR-21 expression in non-cancer and CRC patients. Using the same serum sets from normal and CRC patients, we found that the serum miR-21 levels were significantly increased in the CRC patients when compared to levels in the non-tumor control patients $(\mathrm{P}=0.003)$. AUC of the ROC curves was 0.675 . When a cut-off point was set at 0.0107 , the sensitivity was $54.7 \%$ and the specificity was $84.4 \%$ in discriminating CRC from non-tumor control subjects. CRC, colorectal cancer.

Table I. Relationship between miR-199a-3p and clinicopathological features.

\begin{tabular}{|c|c|c|c|}
\hline & \multicolumn{3}{|c|}{$\operatorname{miR} 199$} \\
\hline & High & Low & $\mathrm{P}$-value \\
\hline Gender & & & 0.354 \\
\hline Male & 30 & 26 & \\
\hline Female & 12 & 16 & \\
\hline Differentiation & & & 0.502 \\
\hline Well & 15 & 18 & \\
\hline Mod, por, muc & 27 & 24 & \\
\hline Tumor size (mm) & & & 0.189 \\
\hline$\geq 35$ & 25 & 19 & \\
\hline$<35$ & 17 & 23 & \\
\hline Serosal invasion & & & $0.022^{\mathrm{a}}$ \\
\hline $\mathrm{T} 1, \mathrm{~T} 2$ & 10 & 20 & \\
\hline $\mathrm{T} 3, \mathrm{~T} 4$ & 32 & 22 & \\
\hline Lymph node metastatis & & & 0.826 \\
\hline Positive & 19 & 18 & \\
\hline Negative & 23 & 24 & \\
\hline Lymphatic invasion & & & 0.275 \\
\hline Positive & 24 & 19 & \\
\hline Negative & 18 & 23 & \\
\hline Venous invasion & & & 0.232 \\
\hline Positive & 10 & 15 & \\
\hline Negative & 32 & 27 & \\
\hline
\end{tabular}

Well, well differentiated adenocarcinoma; mod, moderately differentiated adenocarcinoma; por, poorly differentiated adenocarcinoma;

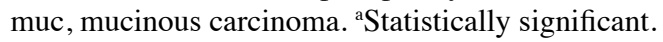

drawn for serum miR-199a-3p, which yielded 0.644 as a value of AUC. When a cut-off point was set at 0.0010 , the sensitivity was $47.6 \%$ and the specificity was $75.0 \%$ in discriminating CRC from the non-tumor control subjects.
miR-199a-3p

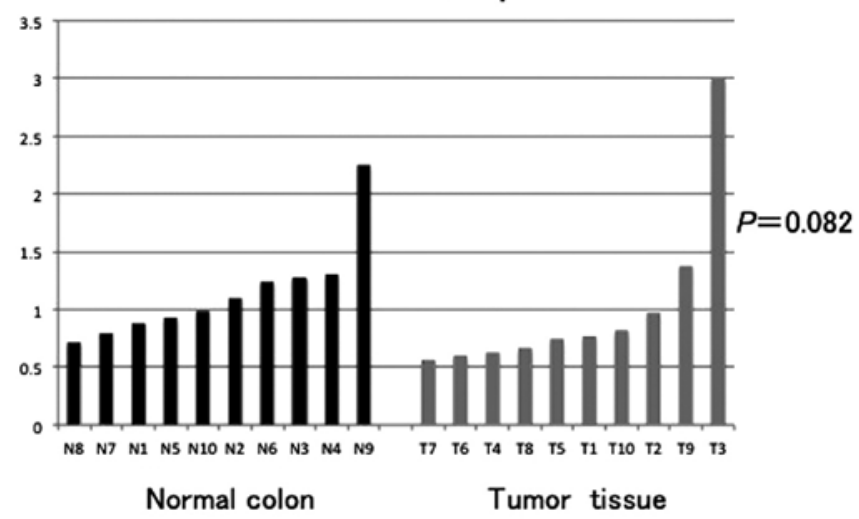

Figure 5. Expression of miR-199a-3p in normal mucosa and CRC tissue samples. We additionally examined miR-199a-3p expression in normal colonic mucosa $(n=10)$ and CRC tissue samples $(n=10)$. There was no significant difference in miR-199a-3p expression $(\mathrm{P}=0.082)$. CRC, colorectal cancer.

Serum miR-21 expression in non-cancer and CRC patients. As a reference, we examined miR-21 as a putative circulating miRNA. Using the same serum sets of normal and CRC patients, we found that the serum miR-21 levels were significantly increased in the CRC patients when compared to levels in the non-tumor control patients $(\mathrm{P}=0.003)$. AUC of the ROC curves was 0.675 . When a cut-off point was set at 0.0107 , the sensitivity was $54.7 \%$ and the specificity was $84.4 \%$ in discriminating CRC from the non-tumor control subjects (Fig. 4).

miR-199a-3p expression in patient serum and clinicopathological characteristics. Clinical and pathological survey indicated that miR-199-3p was significantly associated with deep wall invasion in the high miR-199a-3p patient group when compared to the low miR-199a-3p expression group $(\mathrm{P}=0.022)$, when $\mathrm{CRC}$ patients were divided into two groups by median expression (Table I).

Expression of miR-199a-3p in normal mucosa and CRC tissue samples. We additionally examined miR-199a-3p expression 
in normal colonic mucosa $(\mathrm{n}=10)$ and CRC tissue samples $(\mathrm{n}=10)$. No significant difference in miR-199a-3p expression was noted ( $\mathrm{P}=0.082$, Fig. 5).

\section{Discussion}

In the present study we aimed to identify a novel serum marker for colorectal cancer (CRC) by comparison of the patient serum before and after surgery, using the miRNA array. The miRNAs were mostly reduced after surgery to various extents. Among them, we focused on miR-199a-3p and let-7a since the reduction rate of the miRNAs was relatively large. qRT-PCR verified the results obtained by the miRNA array in an extended number of CRC patients. These findings suggest that the two miRNAs may be derived from the main CRC tumors, either directly or indirectly and that they may be useful to monitor disease progression.

To investigate whether the two miRNAs may be informative as serum tumor markers when compared to non-tumor patients, we compared the serum miRNA expression between non-cancer and CRC patients. We found that expression of miR-199a-3p, but not let-7a, was increased in the CRC patients when compared with the non-tumor patients. Based on these findings, we suggest that miR-199a-3p could be a superior marker for detection of CRC by blood tests. Indeed, miR-199a-3p levels increased going from non-cancer patients to CRC patients and were markedly decreased after tumor resection.

Clinicopathological survey showed that the serum miR199a-3p level was associated with serosal invasion of the primary CRC tumor, suggesting that miR-199a-3p may be associated with tumor invasion. In support of this hypothesis, Wan et al provided evidence that high miR-199a-3p expression in CRC tissue samples contributes to lymph node and liver metastases and advanced TNM stage (18).

In analysis of the tissue-derived miR-199a-3p, we found no significant difference between normal colonic mucosa and CRC tissue samples. These findings suggest that tumor cells may secrete miR-199a-3p to a greater extent than normal epithelial cells, or certain host cells may also contribute to secretion of miR-199a-3p in the tumor-bearing patients. We postulate that the former is more probable since other investigators have reported miR-199a-3p expression in the stool and tumor tissues of CRC patients $(18,19)$. Moreover, it has been shown that anti-miRNA against miR-199a-3p suppressed the growth of colon cancer cells, suggesting that miR-199a-3p is an oncogenic miRNA (18). In spite of these earlier discoveries, we are not aware of any studies reporting that serum miR-199a-3p is a useful biomarker for CRC. Considering the convenience of blood samples that allow monitoring at numerous time points by the relatively easy detection system, our finding of circulating miR-199a-3p in CRC is of clinical importance.

The ROC curve of miR-199a-3p to distinguish cancer patients from non-cancer patients appeared to have limitations in sensitivity and specificity. To estimate its value, we examined serum miR-21 expression in the same series of non-tumor and CRC patients since miR-21 is known as a putative serum biomarker for CRC (17). As a result, we found that the ROC curve of miR-21 was not markedly different of that of miR199a-3p in our patient series.
In conclusion, we identified miR-199a-3p from the differential expression profile between pre-operative and postoperative serum as a novel serum biomarker for CRC. Further investigation of serum miR-199a-3p in regards to patient prognosis and further monitoring of the therapeutic efficacy of chemotherapy are essential.

\section{Acknowledgements}

This study was supported by a Grant-in-Aid for Scientific Research (B) (24390315 to H.Y.)

\section{References}

1. Siegel R, Naishadham D and Jemal A: Cancer statistics, 2013. CA Cancer J Clin 63: 11-30, 2013.

2. Jemel A, Bray F, Center MM, Ferlay J, Ward E and Forman D: Global cancer statistics. CA Cancer J Clin 61: 69-90, 2011.

3. O'Connell JB, Maggard MA and Ko CY: Colon cancer survival rates with the new American Joint Committee on Cancer sixth edition staging. J Natl Cancer Inst 6: 1420-1425, 2004.

4. Hewitson P, Glasziou P, Watson E, Towler B and Irwig L: Cochrane systematic review of colorectal cancer screening using the fecal occult blood test (hemoccult): an update. Am J Gastroenterol 103: 1541-1549, 2008.

5. Walsh JM and Terdiman JP: Colorectal cancer screening: scientific review. JAMA 289: 1288-1296, 2003.

6. Baxter NN, Warren JL, Barrett MJ, Stukel TA and DoriaRose VP: Association between colonoscopy and colorectal cancer mortality in a US cohort according to site of cancer and colonoscopist specialty. J Clin Oncol 30: 2664-2669, 2012.

7. Winawer S, Fletcher R, Rex D, et al: Colorectal cancer screening and surveillance: clinical guidelines and rationale - Update based on new evidence. Gastroenterology 124: 544-560, 2003.

8. He L, Thomson JM, Hemann MT, et al: A microRNA polycistron as a potential human oncogene. Nature 435: 828-833, 2005.

9. Filipowicz W, Bhattacharyya SN and Sonenberg N: Mechanisms of post-transcriptional regulation by microRNAs: are the answers in sight? Nat Rev Genet 9: 102-114, 2008.

10. Schetter AJ, Leung SY, Sohn JJ, et al: MicroRNA expression profiles associated with prognosis and therapeutic outcome in colon adenocarcinoma. JAMA 299: 425-436, 2008.

11. Mitchell PS, Parkin RK, Kroh EM, et al: Circulating microRNAs as stable blood-based markers for cancer detection. Proc Nat Acad Sci USA 105: 10513-10518, 2008.

12. Schwarzenbach H, Hoon DS and Pantel K: Cell-free nucleic acids as biomarkers in cancer patients. Nat Rev Cancer 11: 426-437, 2011.

13. Pu XX, Huang GL, Guo HQ, et al: Circulating miR-221 directly amplified from plasma is a potential diagnostic and prognostic marker of colorectal cancer and is correlated with p53 expression. J Gastroenterol Hepatol 25: 1674-1680, 2010.

14. Ng EK, Chong WW, Jin H, et al: Differential expression of microRNAs in plasma of patients with colorectal cancer: a potential marker for colorectal cancer screening. Gut 58: 1375-1381, 2009.

15. Huang Z, Huang D, Ni S, Peng Z, Sheng W and Du X: Plasma microRNAs are promising novel biomarkers for early detection of colorectal cancer. Int J Cancer 127: 118-126, 2010.

16. Cheng H, Zhang L, Cogdell DE, et al: Circulating plasma miR-141 is a novel biomarker for metastatic colon cancer and predicts poor prognosis. PLoS One 6: e17745, 2010.

17. Toiyama Y, Takahashi M, Hur K, et al: Serum miR-21 as a diagnostic and prognostic biomarker in colorectal cancer. J Natl Cancer Inst 105: 849-859, 2013.

18. Wan D, He S, Xie B, et al: Aberrant expression of miR-199a-3p and its clinical significance in colorectal cancers. Med Oncol 30: 378, 2013.

19. Ahmed FE, Ahmed NC, Vos PW, et al: Diagnostic microRNA markers to screen for sporadic human colon cancer in stool: I. Proof of principle. Cancer Genomics Proteomics 10: 93-113, 2013. 